\title{
Economic Impacts of Agricultural and Natural Resource Industries in Florida, 20031
}

Alan W. Hodges and W. David Mulkey²

Estimates of economic impacts were developed for the agricultural and natural resource industries in Florida in 2003, based on Implan data for Florida, which are derived from the National Income and Product Accounts of the United States and various regional data sources (MIG, 2005). Over 100 individual sectors were identified as related to agriculture and natural resources, including sectors for commodity production, processing and manufacturing, and associated input suppliers and supporting services. However, wholesale and retail distribution of food and kindred products, restaurants, and other food service establishments were excluded from the analysis, since these are not strictly related to agriculture or natural resources. Estimates of total regional economic impacts for each sector were computed using Implan SAM multipliers, including the indirect and induced effects of domestic and foreign export sales outside Florida. Indirect effects represent the impacts of allied businesses that supply inputs to the agricultural and natural resource industries, while induced effects represent the impacts of industry employee household spending. Values were expressed in 2005 U.S. dollars using the Gross Domestic Product (GDP) Implicit Price Deflator (U.S. Department of Commerce), which restated values for 2003 approximately 5.7 percent higher. Results of the analysis are shown in the following tables: Table 1 summarizes the impact estimates by major industry groups; Table 2 provides detailed estimates for individual sectors within each group; Table 3 gives value added impacts in nine economic regions of Florida; Table 4 indicates the change in value added impacts over the period 2001-2003; and Table 5 shows the direct employment and value added by agriculture and natural resources in comparison to all other industry groups in Florida.

Total industry output or sales of the agricultural and natural resource industries in Florida in 2003 was $\$ 50.8$ billion ( $\mathrm{Bn})$, while total output impacts, including the indirect and induced multiplier effects, were $\$ 87.6 \mathrm{Bn}$ (Table 1). Total direct employment was 388,916 jobs, and total employment impacts were 756,993 jobs. Total value added was $\$ 19.2 \mathrm{Bn}$, and total value added impacts were $\$ 41.1 \mathrm{Bn}$, which represents the net value created by the industry, measured as the difference between industry revenues and input purchases from other sectors. It also represents personal and business net income, and capital consumption. The labor (earned) income impact of agriculture and natural resources was \$25.1

1. This is EDIS document FE627, a publication of the Food and Resource Economics Department, Florida Cooperative Extension Service, Institute of Food and Agricultural Sciences, University of Florida, Gainesville, FL. Published February 2006. Please visit the EDIS website at http://edis.ifas.ufl.edu.

2. Alan W. Hodges, Associate-In, and W. David Mulkey, Professor, Food and Resource Economics Department, Florida Cooperative Extension Service, Institute of Food and Agricultural Sciences, University of Florida, Gainesville, FL.

The Institute of Food and Agricultural Sciences (IFAS) is an Equal Opportunity Institution authorized to provide research, educational information and other services only to individuals and institutions that function with non-discrimination with respect to race, creed, color, religion, age, disability, sex, sexual orientation, marital status, national origin, political opinions or affiliations. U.S. Department of Agriculture, Cooperative Extension Service, University of Florida, IFAS, Florida A. \& M. University Cooperative Extension Program, and Boards of County Commissioners Cooperating. Larry Arrington, Dean 
$\mathrm{Bn}$. Indirect business taxes paid to local, state, and federal governments were $\$ 2.6 \mathrm{Bn}$. Total domestic and foreign export sales outside the state of Florida were \$27.6 Bn.

As shown in Table 1, the largest industry groups in terms of value added impacts were fruit and vegetable farming and processing $(\$ 7.5 \mathrm{Bn})$, environmental horticulture $(\$ 6.8 \mathrm{Bn})$, forestry and forest products $(\$ 6.5 \mathrm{Bn})$, agricultural inputs and support services $(\$ 6.5 \mathrm{Bn})$, other food product manufacturing ( $\$ 5.5 \mathrm{Bn})$, tobacco farming and manufacturing ( $\$ 3.0 \mathrm{Bn})$, sugarcane farming and refined sugar manufacturing (\$1.8 Bn), mining (\$1.6 $\mathrm{Bn})$, and livestock and dairy farming and animal products manufacturing $(\$ 1.2 \mathrm{Bn})$. Some other smaller industry groups with value added impacts less than $\$ 1$ billion were fishing and seafood products (\$413 Mn), grain and oilseed farming and processing (\$93 Mn), other crop farming, including cotton, nuts, and miscellaneous crops ( $\$ 239 \mathrm{Mn})$, and wildlife hunting and trapping (\$45 Mn).

As shown in Table 2, some of the largest individual industry sectors, all with over one billion dollars in value added impacts were landscape services ( $\$ 4.16 \mathrm{Bn})$, soft drink and ice manufacturing $(\$ 2.92 \mathrm{Bn})$, other tobacco product manufacturing $(\$ 2.79 \mathrm{Bn})$, greenhouse and nursery production (\$2.66 Bn), phosphatic fertilizer manufacturing (\$2.39 Bn), fruit and vegetable canning and drying $(\$ 2.26 \mathrm{Bn})$, agriculture and forestry support activities $(\$ 2.12 \mathrm{Bn})$, fruit farming $(\$ 1.99 \mathrm{Bn})$, vegetable and melon farming ( $\$ 1.98 \mathrm{Bn})$, frozen food manufacturing ( $\$ 1.27 \mathrm{Bn})$, sugar manufacturing $(\$ 1.26 \mathrm{Bn})$, paper and paperboard mills $(\$ 1.23 \mathrm{Bn})$, and forest nurseries and timber tracts $(\$ 1.05 \mathrm{Bn})$.

Regional value added impacts of agriculture and natural resource industries are shown in Table 3 for the nine economic regions of Florida, defined based on employee commuting patterns. A map of the counties included in these regions is shown in Figure 1. Total value added impacts were largest in the Miami-Ft. Lauderdale region (\$11.84 Bn), followed by Orlando ( $\$ 9.37 \mathrm{Bn}$ ), Tampa-St.Petersburg-Clearwater $(\$ 6.71 \mathrm{Bn})$, Jacksonville ( $\$ 5.70 \mathrm{Bn})$, Sarasota-Bradenton (\$3.78 $\mathrm{Bn})$, Gainesville (\$1.19 Bn), Tallahassee (\$1.21 Bn), Pensacola (\$688 Mn) and Panama City (\$381 Mn).

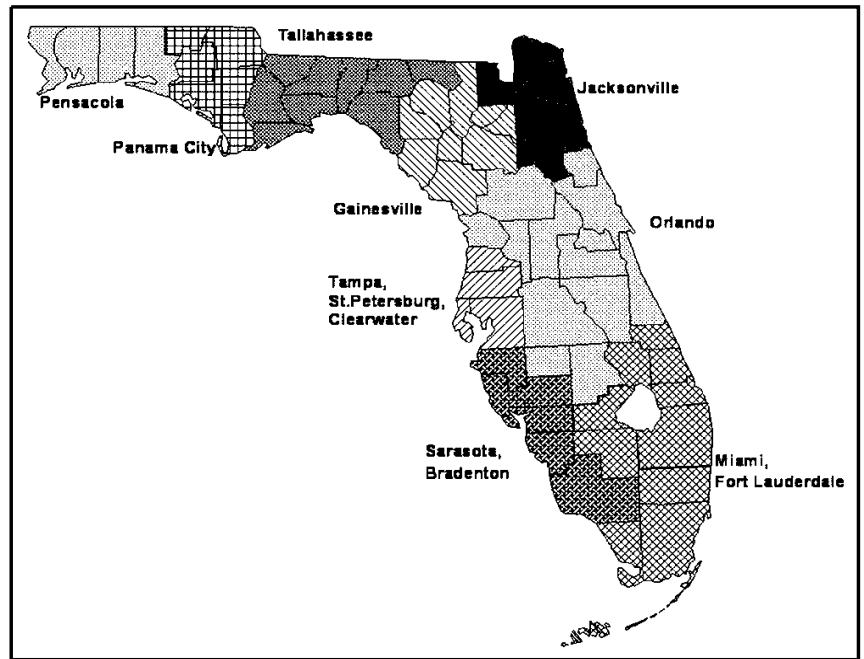

Figure 1. Economic regions of Florida.

The total value added impacts of agricultural and natural resource industries in Florida grew from $\$ 33.77 \mathrm{Bn}$ in 2001 to $\$ 41.12 \mathrm{Bn}$ in 2003 , adjusted for inflation to express in 2005 U.S. dollars (Table 4). This represented an overall growth rate of 20 percent, or 10 percent per annum. Rapid growth occurred for tobacco products (104\%), other crop farming (33\%), and forest products (27\%); moderate growth occurred for environmental horticulture (17\%), fruits and vegetables (17\%), agricultural inputs and supporting services (16\%), and other food product manufacturing (15\%); and negative growth occurred for grains and oilseeds (-93\%), wildlife hunting and trapping (-55\%), and livestock, dairy and animal products $(-13 \%)$. Note that these trends in value added impacts reflect changes in the structure of the Florida economy as well as changes in industry activity and commodity prices.

The relative importance of agricultural and natural resource industries in Florida can be gauged by its share of overall economic activity in the state, as shown in Table 5. The Gross State Product (GSP), which is the sum of value added for all industries, was $\$ 578.9 \mathrm{Bn}$ in 2003 (expressed in 2005 U.S. dollars), and the total employment in the state was 9,391,709 jobs. The direct value added by agriculture and natural resource industries ( $\$ 19.2 \mathrm{Bn}$ ) comprised about 3.3 percent GSP, while direct employment (388,916 jobs) represented 4.1 percent of total state employment. However, the total value added impacts of agricultural and natural resource industries (\$41.1 $\mathrm{Bn}$ ) represented 7.1 percent GSP, and the total employment impact (756,993 jobs) accounted for 8.1 
percent of total state employment. By comparison, the largest industry group in Florida was real estate and financial services, whose direct value added represented 14.2 percent GSP, followed by professional and technical services $(12.7 \%)$, retail trade $(7.8 \%)$, government $(7.2 \%)$, and health care (7.1\%).

\section{References}

Johnson, K., and J. Kort. 2004. Redefinition of the BEA Economic Areas. Survey of Current

Business, pp. 68-75, Bureau of Economic Analysis, U.S. Department of Commerce, Washington, D.C. (November).

MIG, Inc. 2005. Implan data for Florida counties (2003), Minnesota Implan Group, Inc., Stillwater, MN (December). 


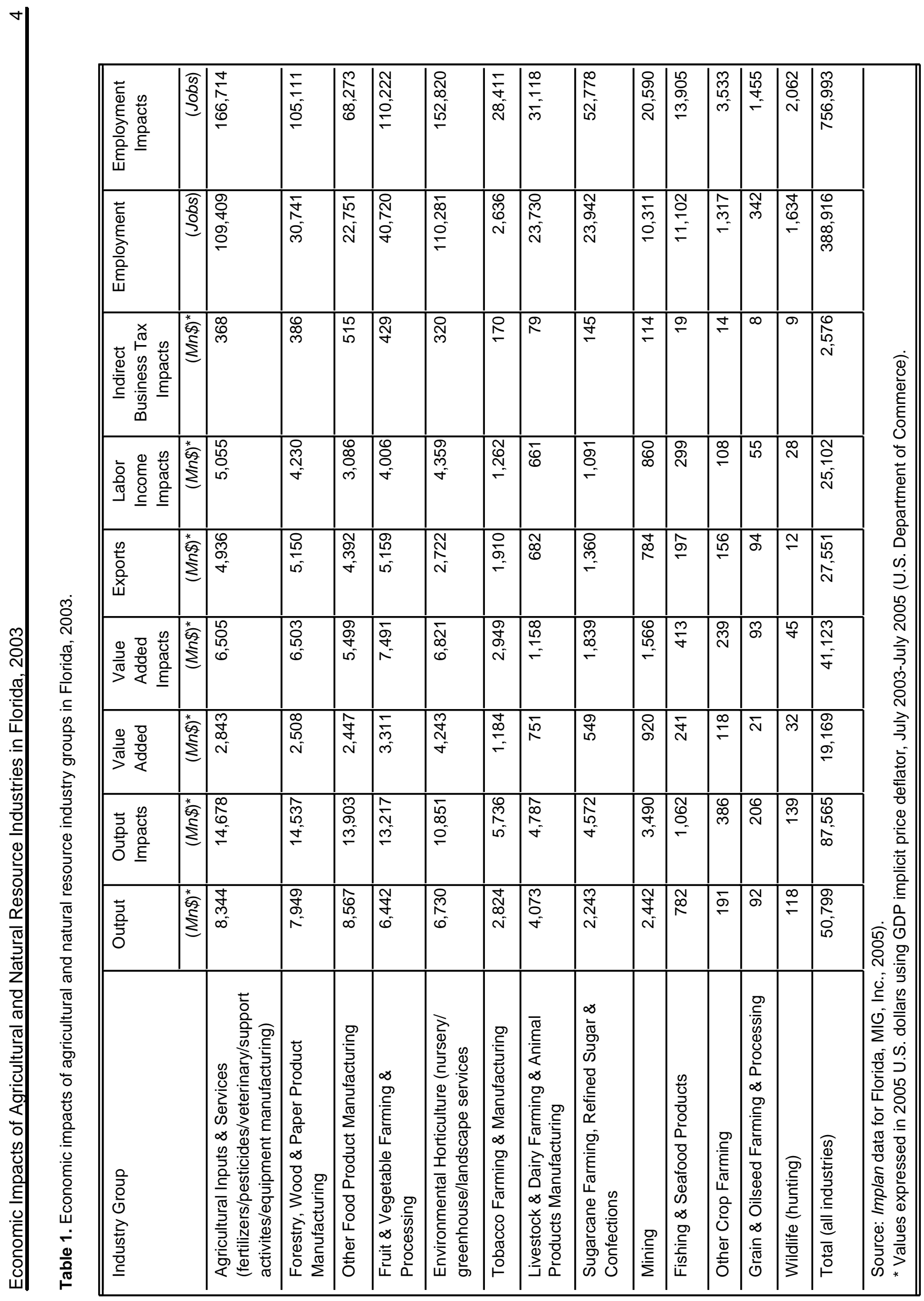


Table 2. Economic impacts of agricultural and natural resource industry sectors in Florida, 2003.

\begin{tabular}{|c|c|c|c|c|c|}
\hline Industry Group & Industry Sector & Output & $\begin{array}{l}\text { Output } \\
\text { Impacts }\end{array}$ & $\begin{array}{l}\text { Employment } \\
\text { Impacts }\end{array}$ & $\begin{array}{l}\text { Value Added } \\
\text { Impacts }\end{array}$ \\
\hline & & $(M n \$)^{*}$ & $(M n \$)^{*}$ & (Jobs) & $(M n \$)^{*}$ \\
\hline \multirow{5}{*}{$\begin{array}{l}\text { Fruit \& Vegetable } \\
\text { Farming \& } \\
\text { Processing }\end{array}$} & Frozen food manufacturing & $1,165.5$ & $2,588.0$ & 18,276 & $1,268.9$ \\
\hline & $\begin{array}{l}\text { Fuirt \& vegetable canning \& } \\
\text { drying }\end{array}$ & $2,110.6$ & $4,663.6$ & 28,498 & $2,258.8$ \\
\hline & Fruit farming & $1,606.1$ & $3,140.3$ & 39,035 & $1,987.8$ \\
\hline & Vegetable \& melon farming & $1,559.7$ & $2,825.1$ & 25,392 & $1,975.9$ \\
\hline & Total & $6,442.0$ & $13,217.0$ & 110,222 & $7,491.4$ \\
\hline \multirow{3}{*}{$\begin{array}{l}\text { Environmental } \\
\text { Horticulture } \\
\text { (nursery/greenhouse/ } \\
\text { landscape services) }\end{array}$} & $\begin{array}{l}\text { Greenhouse \& nursery } \\
\text { production }\end{array}$ & $1,747.5$ & $3,329.7$ & 35,952 & $2,658.5$ \\
\hline & $\begin{array}{l}\text { Landscape services }(70 \% \\
\text { services to bldgs sector) }\end{array}$ & $4,982.8$ & $7,521.8$ & 116,868 & $4,162.2$ \\
\hline & Total & $6,730.3$ & $10,851.4$ & 152,820 & $6,820.8$ \\
\hline \multirow{13}{*}{$\begin{array}{l}\text { Agricultural Inputs \& } \\
\text { Services (fertilizers/ } \\
\text { pesticides/veterinary/ } \\
\text { support activities/ } \\
\text { equipment } \\
\text { manufacturing) }\end{array}$} & $\begin{array}{l}\text { Agriculture \& forestry support } \\
\text { activities }\end{array}$ & $1,505.9$ & $3,035.3$ & 95,204 & $2,118.2$ \\
\hline & $\begin{array}{l}\text { Farm machinery \& equipment } \\
\text { manufacturing }\end{array}$ & 153.0 & 189.0 & 846 & 58.3 \\
\hline & $\begin{array}{l}\text { Fertilizer, mixing only, } \\
\text { manufacturing }\end{array}$ & 285.5 & 285.5 & 790 & 67.2 \\
\hline & $\begin{array}{l}\text { Food product machinery } \\
\text { manufacturing }\end{array}$ & 66.0 & 84.9 & 580 & 33.8 \\
\hline & $\begin{array}{l}\text { Lawn \& garden equipment } \\
\text { manufacturing }\end{array}$ & 15.0 & 16.7 & 54 & 3.3 \\
\hline & $\begin{array}{l}\text { New farm housing units \& } \\
\text { additions \& alterations }\end{array}$ & $1,026.7$ & $1,026.7$ & 4,149 & 334.6 \\
\hline & $\begin{array}{l}\text { Nitrogenous fertilizer } \\
\text { manufacturing }\end{array}$ & 595.4 & $1,077.4$ & 4,492 & $356 / 8$ \\
\hline & $\begin{array}{l}\text { Paper industry machinery } \\
\text { manufacturing }\end{array}$ & 19.7 & 26.9 & 172 & 10.0 \\
\hline & $\begin{array}{l}\text { Pesticide \& other agricultural } \\
\text { chemical manufacturing }\end{array}$ & 421.3 & 859.9 & 4,359 & 388.9 \\
\hline & $\begin{array}{l}\text { Phosphatic fertilizer } \\
\text { manufacturing }\end{array}$ & $3,088.5$ & $6,587.8$ & 34,594 & $2,393.4$ \\
\hline & $\begin{array}{l}\text { Sawmill \& woodworking } \\
\text { machinery }\end{array}$ & 5.0 & 6.1 & 44 & 2.3 \\
\hline & Veterinary services & $1,162.4$ & $1,481.5$ & 21,430 & 738.6 \\
\hline & Total & $8,344.4$ & $14,677.8$ & 166,714 & $6,505.4$ \\
\hline
\end{tabular}


Table 2. Economic impacts of agricultural and natural resource industry sectors in Florida, 2003.

\begin{tabular}{|c|c|c|c|c|c|}
\hline Industry Group & Industry Sector & Output & $\begin{array}{l}\text { Output } \\
\text { Impacts }\end{array}$ & $\begin{array}{l}\text { Employment } \\
\text { Impacts }\end{array}$ & $\begin{array}{l}\text { Value Added } \\
\text { Impacts }\end{array}$ \\
\hline & & $(M n \$)^{*}$ & $(M n \$)^{*}$ & $\overline{(\text { Jobs })}$ & $(M n \$)^{\prime}$ \\
\hline \multirow{21}{*}{$\begin{array}{l}\text { Forestry, Wood, \& } \\
\text { Paper Product } \\
\text { Manufacturing }\end{array}$} & $\begin{array}{l}\text { All other converted paper } \\
\text { product manufacturing }\end{array}$ & 43.2 & 101.2 & 730 & 50.3 \\
\hline & $\begin{array}{l}\text { Coated \& laminated paper \& } \\
\text { packaging materials }\end{array}$ & 142.3 & 319.8 & 2,081 & 148.6 \\
\hline & $\begin{array}{l}\text { Coated \& uncoated paper bag } \\
\text { manufacturing }\end{array}$ & 132.9 & 264.8 & 1,718 & 112.6 \\
\hline & $\begin{array}{l}\text { Cut stock, resawing lumber, \& } \\
\text { planing }\end{array}$ & 18.9 & 29.3 & 238 & 10.5 \\
\hline & $\begin{array}{l}\text { Engineered wood member \& } \\
\text { truss manufacturing }\end{array}$ & 930.1 & $1,443.8$ & 10,771 & 679.3 \\
\hline & $\begin{array}{l}\text { Forest nurseries, forest } \\
\text { products \& timber }\end{array}$ & 578.4 & $1,856.6$ & 27,022 & $1,046.6$ \\
\hline & Logging & 605.8 & 678.7 & 3,695 & 299.8 \\
\hline & $\begin{array}{l}\text { Miscellaneous wood product } \\
\text { manufacturing }\end{array}$ & 55.3 & 61.5 & 540 & 24.6 \\
\hline & $\begin{array}{l}\text { Other millwork, including } \\
\text { flooring }\end{array}$ & 288.1 & 299.7 & 1,878 & 83.3 \\
\hline & Paper \& paperboard mills & $1,058.3$ & $2,604.3$ & 15,911 & $1,228.3$ \\
\hline & $\begin{array}{l}\text { Paperboard container } \\
\text { manufacturing }\end{array}$ & $1,136.1$ & $2,023.3$ & 12,379 & 815.1 \\
\hline & Pulp mills & 723.6 & $1,882.6$ & 11,573 & 818.0 \\
\hline & $\begin{array}{l}\text { Reconstituted wood product } \\
\text { manufacturing }\end{array}$ & 3.0 & 3.8 & 17 & 1.6 \\
\hline & $\begin{array}{l}\text { Sanitary paper product } \\
\text { manufacturing }\end{array}$ & 738.7 & $1,378.6$ & 7,630 & 667.9 \\
\hline & Sawmills & 465.4 & 518.5 & 2,444 & 147.1 \\
\hline & $\begin{array}{l}\text { Surface-coated paperboard } \\
\text { manufacturing }\end{array}$ & 8.3 & 9.1 & 35 & 2.3 \\
\hline & $\begin{array}{l}\text { Veneer \& plywood } \\
\text { manufacturing }\end{array}$ & 193.5 & 218.7 & 1,356 & 71.7 \\
\hline & $\begin{array}{l}\text { Wood container \& pallet } \\
\text { manufacturing }\end{array}$ & 153.7 & 158.1 & 1,475 & 64.3 \\
\hline & Wood preservation & 156.3 & 159.6 & 635 & 35.0 \\
\hline & $\begin{array}{l}\text { Wood windows \& door } \\
\text { manufacturing }\end{array}$ & 516.9 & 524.8 & 2,982 & 196.5 \\
\hline & Total & $7,948.7$ & $14,536.7$ & 105,111 & $6,503.3$ \\
\hline
\end{tabular}


Economic Impacts of Agricultural and Natural Resource Industries in Florida, 2003

Table 2. Economic impacts of agricultural and natural resource industry sectors in Florida, 2003.

\begin{tabular}{|c|c|c|c|c|c|}
\hline Industry Group & Industry Sector & Output & $\begin{array}{l}\text { Output } \\
\text { Impacts }\end{array}$ & $\begin{array}{l}\text { Employment } \\
\text { Impacts }\end{array}$ & $\begin{array}{l}\text { Value Added } \\
\text { Impacts }\end{array}$ \\
\hline & & $(M n \$)^{*}$ & $(M n \$)^{*}$ & (Jobs) & $(M n \$)^{*}$ \\
\hline \multirow{22}{*}{$\begin{array}{l}\text { Other Food Product } \\
\text { Manufacturing }\end{array}$} & All other food manufacturing & 121.6 & 141.1 & 652 & 32.2 \\
\hline & $\begin{array}{l}\text { Bread \& bakery product, } \\
\text { except frozen, manufacturing }\end{array}$ & $1,166.1$ & $1,180.0$ & 9,786 & 535.4 \\
\hline & $\begin{array}{l}\text { Breakfast cereal } \\
\text { manufacturing }\end{array}$ & 0.0 & 0.0 & 0 & 0.0 \\
\hline & Breweries & 787.3 & $1,615.2$ & 8.943 & 880.4 \\
\hline & Coffee \& tea manufacturing & 270.3 & 298.4 & 800 & 63.7 \\
\hline & $\begin{array}{l}\text { Cookie } \& \text { cracker } \\
\text { manufacturing }\end{array}$ & 290.3 & 290.3 & 914 & 89.2 \\
\hline & Distilleries & 239.2 & 524.3 & 3,404 & 341.5 \\
\hline & Dog \& cat food manufacturing & 29.5 & 31.2 & 47 & 2.6 \\
\hline & Dry pasta manufacturing & 11.9 & 12.4 & 49 & 3.2 \\
\hline & Fats $\&$ oils refining $\&$ blending & 41.8 & 57.9 & 179 & 15.0 \\
\hline & $\begin{array}{l}\text { Flavoring syrup \& concentrate } \\
\text { manufacturing }\end{array}$ & 631.3 & 647.8 & 479 & 121.7 \\
\hline & $\begin{array}{l}\text { Frozen cakes \& other pastries } \\
\text { manufacturing }\end{array}$ & 6.8 & 7.4 & 65 & 2.1 \\
\hline & $\begin{array}{l}\text { Mayonnaise, dressing, \& } \\
\text { sauce manufacturing }\end{array}$ & 156.6 & 168.6 & 508 & 51.1 \\
\hline & $\begin{array}{l}\text { Mixes \& dough made from } \\
\text { purchased flour }\end{array}$ & 0.6 & 0.6 & 2 & 0.1 \\
\hline & $\begin{array}{l}\text { Other animal food } \\
\text { manufacturing }\end{array}$ & 304.1 & 538.1 & 2,523 & 167.3 \\
\hline & $\begin{array}{l}\text { Other snack food } \\
\text { manufacturing }\end{array}$ & 414.8 & 422.1 & 877 & 131.0 \\
\hline & $\begin{array}{l}\text { Roasted uts \& peanut butter } \\
\text { manufacturing }\end{array}$ & 4.6 & 5.9 & 23 & 1.9 \\
\hline & $\begin{array}{l}\text { Soft drink \& ice } \\
\text { manufacturing }\end{array}$ & $3,751.3$ & $7,568.7$ & 37,696 & $2,915.6$ \\
\hline & $\begin{array}{l}\text { Spice \& extract } \\
\text { manufacturing }\end{array}$ & 284.9 & 293.3 & 703 & 103.7 \\
\hline & Tortilla manufacturing & 0.1 & 0.1 & 1 & 0.0 \\
\hline & Wineries & 54.5 & 100.1 & 622 & 41.4 \\
\hline & Total & $8,567.5$ & $13,903.3$ & 68,273 & $5,499.0$ \\
\hline
\end{tabular}


Table 2. Economic impacts of agricultural and natural resource industry sectors in Florida, 2003.

\begin{tabular}{|c|c|c|c|c|c|}
\hline Industry Group & Industry Sector & Output & $\begin{array}{l}\text { Output } \\
\text { Impacts }\end{array}$ & $\begin{array}{l}\text { Employment } \\
\text { Impacts }\end{array}$ & $\begin{array}{l}\text { Value Added } \\
\text { Impacts }\end{array}$ \\
\hline & & $(M n \$)^{\star}$ & $(M n \$)^{*}$ & (Jobs) & $(M n \$)^{\prime}$ \\
\hline \multirow{5}{*}{$\begin{array}{l}\text { Tobacco Farming \& } \\
\text { Manufacturing }\end{array}$} & Cigarette manufacturing & 236.1 & 248.8 & 229 & 119.8 \\
\hline & $\begin{array}{l}\text { Other tobacco product } \\
\text { manufacturing }\end{array}$ & $2,545.0$ & $5,408.1$ & 27,306 & $2,793.2$ \\
\hline & Tobacco farming & 20.7 & 43.7 & 701 & 28.6 \\
\hline & $\begin{array}{l}\text { Tobacco stemming \& } \\
\text { redrying }\end{array}$ & 22.2 & 35.6 & 177 & 8.0 \\
\hline & Total & $2,823.9$ & $5,736.1$ & 28,411 & $2,949.5$ \\
\hline \multirow{6}{*}{$\begin{array}{l}\text { Sugarcane Farming, } \\
\text { Refined Sugar \& } \\
\text { Confections }\end{array}$} & $\begin{array}{l}\text { Confectionery manufacturing } \\
\text { from cacao beans }\end{array}$ & 10.1 & 12.4 & 44 & 3.7 \\
\hline & $\begin{array}{l}\text { Confectionery manufacturing } \\
\text { from purchased chocolate }\end{array}$ & 114.8 & 159.8 & 878 & 51.4 \\
\hline & $\begin{array}{l}\text { Nonchocolate confectionery } \\
\text { manufacturing }\end{array}$ & 138.8 & 198.9 & 1,120 & 83.9 \\
\hline & Sugar manufacturing & $1,390.2$ & $3,302.4$ & 26,645 & $1,263.6$ \\
\hline & $\begin{array}{l}\text { Sugarcane \& sugar beet } \\
\text { farming }\end{array}$ & 588.9 & 898.7 & 24,091 & 436.4 \\
\hline & Total & $2,242.8$ & $4,572.2$ & 52,778 & $1,839.0$ \\
\hline \multirow[t]{12}{*}{ Mining } & Coal mining & 9.8 & 14.6 & 78 & 7.8 \\
\hline & $\begin{array}{l}\text { Copper, nickel, lead, \& zinc } \\
\text { mining }\end{array}$ & 0.4 & $0 . .5$ & 4 & 0.3 \\
\hline & Drilling oil \& gas wells & 22.2 & 47.2 & 479 & 28.4 \\
\hline & $\begin{array}{l}\text { Gold, silver, \& other metal ore } \\
\text { mining }\end{array}$ & 88.9 & 168.3 & 1,117 & 113.5 \\
\hline & Iron ore mining & 0.4 & 1.0 & 7 & 0.6 \\
\hline & Oil \& gas extraction & $1,456.0$ & $1,485.4$ & 5,193 & 276.1 \\
\hline & $\begin{array}{l}\text { Other nonmetallic mineral } \\
\text { mining }\end{array}$ & 449.2 & 780.8 & 5,513 & 498.3 \\
\hline & $\begin{array}{l}\text { Sand, gravel, clay, \& refractory } \\
\text { mining }\end{array}$ & 145.7 & 373.9 & 3,198 & 247.8 \\
\hline & Stone mining \& quarrying & 253.1 & 580.9 & 4,597 & 367.9 \\
\hline & $\begin{array}{l}\text { Support activities for oil \& gas } \\
\text { operations }\end{array}$ & 11.3 & 23.3 & 288 & 17.2 \\
\hline & $\begin{array}{l}\text { Support activities for other } \\
\text { mining }\end{array}$ & 5.4 & 13.8 & 115 & 8.6 \\
\hline & Total & $2,442.3$ & $3,489.8$ & 20,590 & $1,566.5$ \\
\hline
\end{tabular}


Table 2. Economic impacts of agricultural and natural resource industry sectors in Florida, 2003.

\begin{tabular}{|c|c|c|c|c|c|}
\hline Industry Group & Industry Sector & Output & $\begin{array}{l}\text { Output } \\
\text { Impacts }\end{array}$ & $\begin{array}{l}\text { Employment } \\
\text { Impacts }\end{array}$ & $\begin{array}{l}\text { Value Added } \\
\text { Impacts }\end{array}$ \\
\hline & & $(M n \$)^{\star}$ & $(M n \$)^{\star}$ & $\overline{\text { (Jobs) }}$ & $(M n \$)^{*}$ \\
\hline \multirow{13}{*}{$\begin{array}{l}\text { Livestock \& Dairy } \\
\text { Farming \& Animal } \\
\text { Products } \\
\text { Manufacturing }\end{array}$} & $\begin{array}{l}\text { Animal production, except } \\
\text { cattle \& poultry }\end{array}$ & 267.3 & 318.5 & 8,176 & 77.2 \\
\hline & $\begin{array}{l}\text { Animal, except poultry, } \\
\text { slaughtering }\end{array}$ & 415.7 & 480.3 & 1,863 & 81.0 \\
\hline & Cattle ranching \& farming & 715.5 & $1,044.9$ & 10,871 & 266.0 \\
\hline & Cheese manufacturing & 69.8 & 70.3 & 110 & 5.6 \\
\hline & $\begin{array}{l}\text { Creamery butter } \\
\text { manufacturing }\end{array}$ & 2.7 & 2.8 & 6 & 0.2 \\
\hline & $\begin{array}{l}\text { Dry, condensed, \& evaporated } \\
\text { dairy products }\end{array}$ & 93.8 & 105.1 & 232 & 27.5 \\
\hline & Fluid milk manufacturing & 694.1 & 696.1 & 1,229 & 88.4 \\
\hline & $\begin{array}{l}\text { Ice cream \& frozen dessert } \\
\text { manufacturing }\end{array}$ & 275.3 & 277.7 & 677 & 62.4 \\
\hline & $\begin{array}{l}\text { Meat processed from } \\
\text { carcasses }\end{array}$ & 550.4 & 567.8 & 1,691 & 78.6 \\
\hline & Poultry \& egg production & 376.1 & 551.7 & 2,710 & 284.0 \\
\hline & Poultry processing & 527.6 & 552.1 & 3,104 & 147.1 \\
\hline & $\begin{array}{l}\text { Rendering \& meat by-product } \\
\text { processing }\end{array}$ & 84.4 & 119.8 & 448 & 40.3 \\
\hline & Total & $4,072.9$ & $4,787.1$ & 31,118 & $1,158.4$ \\
\hline \multirow{3}{*}{$\begin{array}{l}\text { Fishing \& Seafood } \\
\text { Products }\end{array}$} & Fishing & 142.2 & 320.3 & 10,263 & 209.6 \\
\hline & $\begin{array}{l}\text { Seafood product preparation \& } \\
\text { packaging }\end{array}$ & 639.6 & 742.1 & 3,642 & 203.6 \\
\hline & Total & 781.8 & $1,062.4$ & 13,905 & 413.2 \\
\hline \multirow[t]{4}{*}{ Other Crop Farming } & All other crop farming & 147.4 & 287.3 & 2,499 & 180.2 \\
\hline & Cotton farming & 42.2 & 95.4 & 998 & 56.7 \\
\hline & Tree nut farming & 1.6 & 3.3 & 36 & 2.1 \\
\hline & Total & 191.2 & 386.0 & 3,533 & 239.0 \\
\hline
\end{tabular}


Table 2. Economic impacts of agricultural and natural resource industry sectors in Florida, 2003.

\begin{tabular}{|c|c|c|c|c|c|c|}
\hline \multirow[t]{2}{*}{ Industry Group } & \multicolumn{2}{|l|}{ Industry Sector } & \multirow{2}{*}{$\begin{array}{l}\text { Output } \\
\frac{(M n \$)^{*}}{}\end{array}$} & \multirow{2}{*}{$\begin{array}{l}\begin{array}{l}\text { Output } \\
\text { Impacts }\end{array} \\
(M n \$)^{*}\end{array}$} & \multirow{2}{*}{$\begin{array}{l}\begin{array}{l}\text { Employment } \\
\text { Impacts }\end{array} \\
\text { (Jobs) }\end{array}$} & \multirow{2}{*}{$\begin{array}{l}\begin{array}{c}\text { Value Added } \\
\text { Impacts }\end{array} \\
(M n \$)\end{array}$} \\
\hline & & & & & & \\
\hline \multirow{5}{*}{$\begin{array}{l}\text { Grain \& Oilseed } \\
\text { Farming \& } \\
\text { Processing }\end{array}$} & Flour milling & & 62.2 & 116.5 & 584 & 46.2 \\
\hline & Grain farming & & 9.1 & 18.9 & 304 & 10.7 \\
\hline & Oilseed farming & & 2.3 & 34.7 & 375 & 21.4 \\
\hline & Rice milling & & 18.8 & 36.3 & 192 & 14.4 \\
\hline & & Total & 92.5 & 206.4 & 1,455 & 92.8 \\
\hline Wildlife (hunting) & & Total & 118.2 & 138.9 & 2,062 & 45.1 \\
\hline Total (all industries) & & & $50,798.5$ & $87,565.1$ & 756,993 & $41,123.3$ \\
\hline
\end{tabular}




\begin{tabular}{|c|c|c|c|c|c|c|c|c|c|c|c|c|c|c|c|c|c|}
\hline & 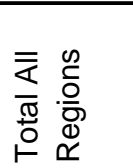 & 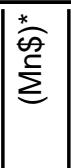 & 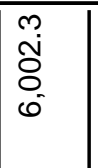 & 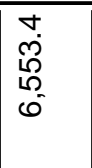 & 离 & \begin{tabular}{|c|}
0 \\
1 \\
0 \\
0 \\
10 \\
10
\end{tabular} & \begin{tabular}{l}
\multirow{2}{*}{} \\
$\stackrel{N}{N}$ \\
\end{tabular} & $\begin{array}{l}1 \\
\dot{0} \\
\infty\end{array}$ & $\begin{array}{l}\stackrel{O}{\mathrm{O}} \\
\stackrel{\mathrm{j}}{\mathrm{j}} \\
\dot{-}\end{array}$ & $\begin{array}{l}\frac{0}{6} \\
\frac{10}{n} \\
\text { is }\end{array}$ & 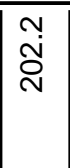 & \begin{tabular}{|c|} 
\\
0 \\
0 \\
0 \\
0 \\
0
\end{tabular} & 每 & 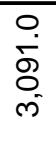 & 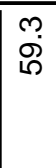 & $\begin{array}{l}0 \\
0 \\
0 \\
0 \\
q\end{array}$ & \\
\hline & $\begin{array}{l}\frac{\pi}{0} \\
\mathbb{0} \\
\mathbb{W} \\
\frac{0}{0} \\
0\end{array}$ & \begin{tabular}{l}
\multirow{2}{*}{} \\
$\stackrel{*}{\check{E}}$ \\
$\Sigma$
\end{tabular} & $\stackrel{+}{\stackrel{亠}{+}}$ & 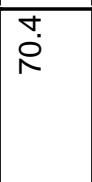 & \begin{tabular}{|c|c|}
$\infty$ \\
$\infty$
\end{tabular} & $\begin{array}{l}0 \\
\dot{0} \\
\dot{0}\end{array}$ & $\begin{array}{l} \\
\\
\text { in }\end{array}$ & $\begin{array}{l}\infty \\
\sim \\
\sim\end{array}$ & $\begin{array}{l}m \\
0 \\
0 \\
\text { in }\end{array}$ & ִָ & 审 & \begin{tabular}{|l}
$\underset{\Gamma}{\Gamma}$ \\
\end{tabular} & $\begin{array}{l}+ \\
0\end{array}$ & $\stackrel{\circ}{\circ}$ & O & $\begin{array}{l}\infty \\
\& \\
0 \\
0\end{array}$ & \\
\hline & 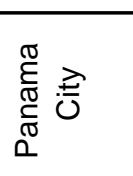 & 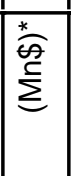 & $\begin{array}{l}\hat{\infty} \\
\infty\end{array}$ & $\begin{array}{l}\infty \\
\stackrel{\infty}{\forall}\end{array}$ & $\bar{\circ}$ & $\begin{array}{l}\mathcal{N} \\
\stackrel{N}{\sigma} \\
\stackrel{5}{*}\end{array}$ & $\stackrel{\sim}{\dot{J}}$ & $\hat{\mu}$ & \begin{tabular}{|l|l}
0 \\
$\dot{9}$
\end{tabular} & $\stackrel{\text { S }}{\mathrm{N}}$ & \begin{tabular}{|l|}
\multirow{2}{*}{} \\
\end{tabular} & $\check{F}$ & m. & $\stackrel{\circ}{\circ}$ & ○. & 品 & \\
\hline ס & 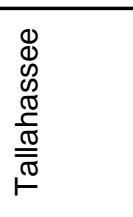 & 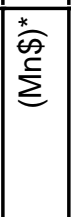 & 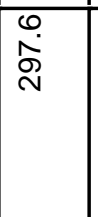 & 占 & 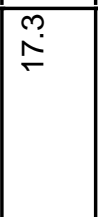 & 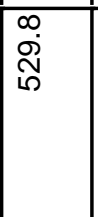 & Nִ & \begin{tabular}{|l|} 
\\
0 \\
0
\end{tabular} & $\begin{array}{l} \\
\\
\circ\end{array}$ & $\bar{i}$ & \begin{tabular}{|l|} 
\\
\end{tabular} & 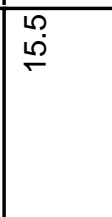 & $\stackrel{\circ}{\circ}$ & $\stackrel{N}{N}$ & $\begin{array}{l}0 \\
\dot{0}\end{array}$ & $\stackrel{m}{\stackrel{m}{N}}$ & 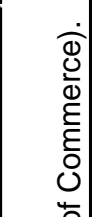 \\
\hline $\begin{array}{l}\text { N } \\
\frac{\delta}{00} \\
\frac{0}{0} \\
\frac{0}{4} \\
\frac{1}{0} \\
0\end{array}$ & 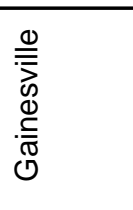 & 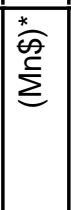 & $\begin{array}{l}+ \\
\dot{0} \\
\stackrel{0}{n}\end{array}$ & $\begin{array}{l}\infty \\
\dot{\infty} \\
\varnothing\end{array}$ & $\begin{array}{l}\dot{\omega} \\
\sim\end{array}$ & 芦 & 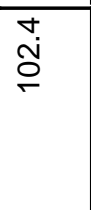 & $\stackrel{N}{N}$ & $\begin{array}{l}0 \\
\dot{+} \\
\dot{q}\end{array}$ & ৯̀. & ষ্ল & $\stackrel{\infty}{\mp}$ & $\stackrel{\circ}{\circ}$ & $\begin{array}{l}\text { ㅇ․ } \\
\stackrel{1}{r}\end{array}$ & $\stackrel{ }{\stackrel{ }{ }}$ & $\begin{array}{l}\infty \\
\infty \\
\stackrel{-}{-}\end{array}$ & 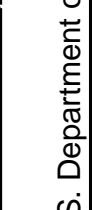 \\
\hline 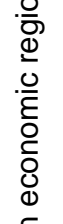 & 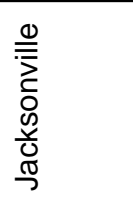 & \begin{tabular}{|l|}
\multirow{2}{*}{} \\
$\stackrel{*}{E}$ \\
$\sum$
\end{tabular} & \begin{tabular}{|l|}
$\stackrel{\rho}{O}$ \\
$\stackrel{S}{\sim}$ \\
\end{tabular} & 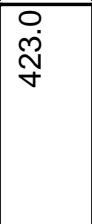 & \begin{tabular}{|l|} 
\\
$\dot{\infty}$ \\
\end{tabular} & 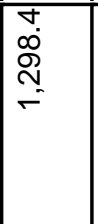 & \begin{tabular}{|l|}
0 \\
0 \\
10 \\
1
\end{tabular} & $\stackrel{\infty}{-}$ & \begin{tabular}{|l|}
$\begin{array}{r}3 \\
0 \\
0\end{array}$ \\
\end{tabular} & $\begin{array}{l}\circ \\
\stackrel{0}{0} \\
\stackrel{0}{\circ}\end{array}$ & $\stackrel{+}{\circ}$ & 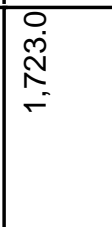 & 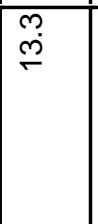 & 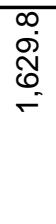 & $\stackrel{m}{\sim}$ & $\begin{array}{l}\infty \\
8 \\
6 \\
15\end{array}$ & 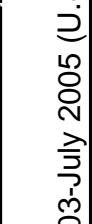 \\
\hline $\begin{array}{l}\overline{0} \\
0 \\
0 \\
0 \\
0 \\
\frac{1}{0} \\
\frac{1}{0} \\
0\end{array}$ & 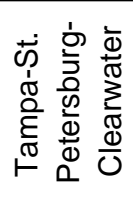 & \begin{tabular}{|l|}
\multirow{2}{*}{} \\
$\stackrel{*}{\mathscr{E}}$ \\
$\sum$
\end{tabular} & $\begin{array}{l}\infty \\
\text { ஸ̃ } \\
\text { ô }\end{array}$ & 㐫 & $\begin{array}{l}\hat{N} \\
\infty \\
\stackrel{N}{N}\end{array}$ & $\begin{array}{l} \\
\dot{0} \\
\dot{\delta} \\
\text { in }\end{array}$ & $\begin{array}{l}\infty \\
\stackrel{\infty}{N} \\
\stackrel{N}{N}\end{array}$ & $\frac{0}{\dot{N}}$ & 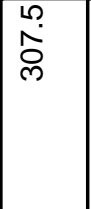 & $\begin{array}{l}\text { : } \\
\text { مे }\end{array}$ & 숭 & \begin{tabular}{|l} 
\\
$\stackrel{O}{D}$ \\
$\stackrel{N}{-}$ \\
$=$
\end{tabular} & Nָ & $\begin{array}{l}0 \\
\dot{0} \\
\infty \\
\infty\end{array}$ & \begin{tabular}{|l|} 
\\
$\dot{+}$ \\
$\dot{+}$
\end{tabular} & $\begin{array}{l}\frac{0}{\lambda} \\
0^{\circ}\end{array}$ & 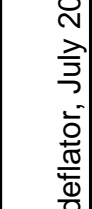 \\
\hline 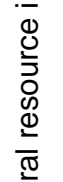 & 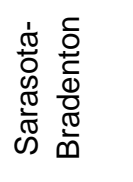 & 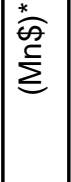 & 柏 & ํㅗㅀ & ĩ & 总 & 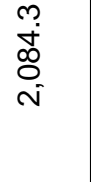 & $\stackrel{\text { Na }}{\longrightarrow}$ & $\underset{\dot{\infty}}{\dot{\infty}}$ & 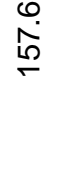 & $\begin{array}{l}0 \\
\dot{m}\end{array}$ & $\begin{array}{l}0 \\
\dot{\tilde{D}}\end{array}$ & $\begin{array}{l}\qquad 0 \\
6 \\
\oplus\end{array}$ & $\stackrel{L}{\infty}$ & $\stackrel{\cong}{=}$ & 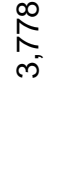 & 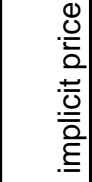 \\
\hline $\begin{array}{l}\frac{\pi}{\pi} \\
\frac{\pi}{0} \\
\frac{0}{\pi} \\
\frac{\pi}{\pi} \\
\frac{\widetilde{J}}{J}\end{array}$ & $\begin{array}{l}\frac{0}{0} \\
\frac{\pi}{0} \\
\frac{\pi}{2}\end{array}$ & 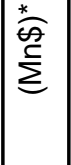 & $\begin{array}{l}\hat{0} \\
\dot{0} \\
\hat{\omega} \\
\text { ì }\end{array}$ & $\begin{array}{l}\stackrel{L}{0} \\
\stackrel{N}{N} \\
\end{array}$ & 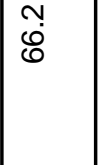 & $\frac{O}{N}$ & 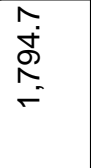 & $\stackrel{\sim}{\dot{\sigma}}$ & $\begin{array}{l}m \\
\text { Dे } \\
\text { D }\end{array}$ & $\frac{⿱}{+}$ & $\begin{array}{l}\dot{j} \\
\ddot{m}\end{array}$ & 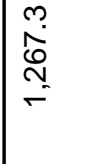 & 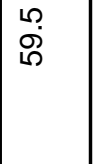 & O. & $\begin{array}{l}m \\
0 \\
0\end{array}$ & $\begin{array}{l}\mathbb{N} \\
\text { m } \\
\sigma\end{array}$ & 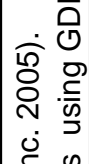 \\
\hline 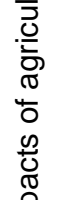 & 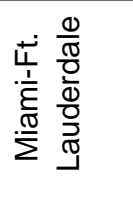 & \begin{tabular}{|l|}
\multirow{2}{*}{} \\
$\stackrel{*}{\mathscr{E}}$ \\
$\sum$
\end{tabular} & $\begin{array}{l}0 \\
\dot{0} \\
\underline{m} \\
\stackrel{-}{-}\end{array}$ & 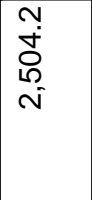 & \begin{tabular}{|l|l}
0 \\
$\frac{m}{\sigma}$ \\
\end{tabular} & $\begin{array}{l}\infty \\
\dot{\omega} \\
\stackrel{N}{N} \\
-\end{array}$ & $\begin{array}{l}\frac{N}{N} \\
\stackrel{N}{N} \\
\text { N }\end{array}$ & $\overline{\bar{Y}}$ & $\begin{array}{l} \\
\dot{D} \\
\dot{\sim}\end{array}$ & 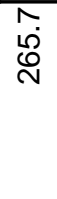 & \begin{tabular}{|l|}
0 \\
+ \\
+
\end{tabular} & $\begin{array}{l}1 \\
8 \\
0 \\
0 \\
0 \\
-\end{array}$ & $\begin{array}{l}\stackrel{j}{0} \\
\stackrel{0}{0} \\
\infty \\
- \\
-\end{array}$ & $\begin{array}{l}\infty \\
0 \\
10 \\
10\end{array}$ & \begin{tabular}{|l|}
\multirow{2}{N}{} \\
$\stackrel{\omega}{\omega}$
\end{tabular} & $\begin{array}{l}\text { J } \\
\infty \\
E \\
F\end{array}$ & 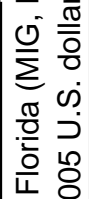 \\
\hline $\begin{array}{l}\frac{.}{0} \\
0 \\
\frac{0}{0} \\
\frac{0}{\pi} \\
0 \\
\frac{0}{10} \\
\frac{\pi}{\pi} \\
m \\
0 \\
\frac{0}{0} \\
\frac{0}{10}\end{array}$ & 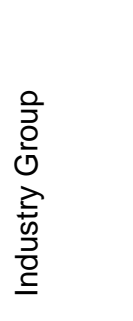 & & 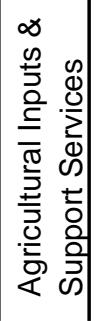 & 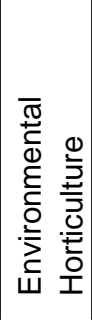 & 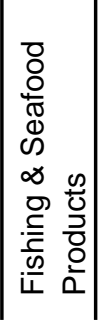 & 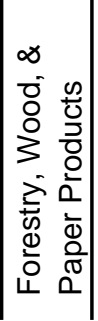 & 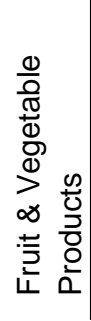 & 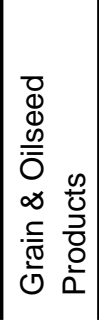 & 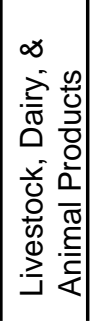 & 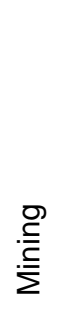 & 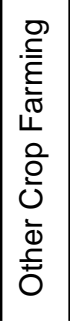 & 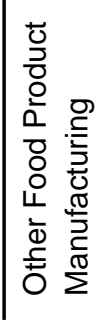 & 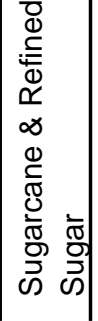 & 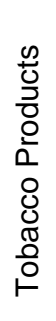 & 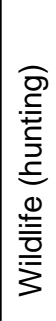 & 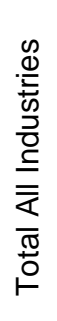 & 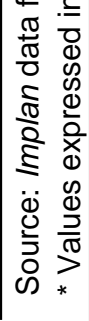 \\
\hline
\end{tabular}


Table 4. Trend in value added impacts of agricultural and natural resource industry groups in Florida, 2001-2003.

\begin{tabular}{|c|c|c|c|c|}
\hline \multirow[t]{2}{*}{ Industry Group } & 2001 & 2002 & 2003 & $\begin{array}{l}\text { Percent } \\
\text { Change } \\
2001-03 \\
\end{array}$ \\
\hline & $(\mathrm{Mn} \$)^{*}$ & $(\mathrm{Mn} \$)^{*}$ & $(\mathrm{Mn} \$)^{*}$ & (\%) \\
\hline $\begin{array}{l}\text { Agricultural Inputs \& Services (fertilizers/pesticides/ } \\
\text { veterinary/support activities/equipment manufacturing }\end{array}$ & 5,559 & 5,631 & 6,503 & $16 \%$ \\
\hline $\begin{array}{l}\text { Environmental Horticulture (nursery/greenhouse/ } \\
\text { landscape services) }\end{array}$ & 5,763 & 6,391 & 6,821 & $17 \%$ \\
\hline Fishing \& Seafood Products & 394 & 436 & 413 & $5 \%$ \\
\hline Forestry, Wood, \& Paper Product Manufacturing & 4,930 & 5,847 & 6,503 & $27 \%$ \\
\hline Fruit \& Vegetable Farming \& Processing & 6,294 & 7,634 & 7,491 & $17 \%$ \\
\hline Grain \& Oilseed Farming \& Processing & 221 & 103 & 93 & $-93 \%$ \\
\hline $\begin{array}{l}\text { Livestock \& Dairy Farming \& Animal Products } \\
\text { Manufacturing }\end{array}$ & 1,315 & 1,110 & 1,158 & $-13 \%$ \\
\hline Mining & 1,445 & 1,533 & 1,566 & $8 \%$ \\
\hline Other Crop Farming & 173 & 190 & 239 & $33 \%$ \\
\hline Other Food Product Manufacturing & 4,752 & 4,850 & 5,499 & $15 \%$ \\
\hline Sugarcane Farming, Refined Sugar \& Confections & 1,678 & 1,688 & 1,839 & $9 \%$ \\
\hline Tobacco Farming \& Manufacturing & 1,172 & 995 & 2,949 & $104 \%$ \\
\hline Wildlife (hunting) & 78 & 54 & 45 & $-55 \%$ \\
\hline Grand Total & 33,774 & 36,462 & 41,120 & $20 \%$ \\
\hline
\end{tabular}


Table 5. Employment and value added of all industry groups in Florida, 2003.

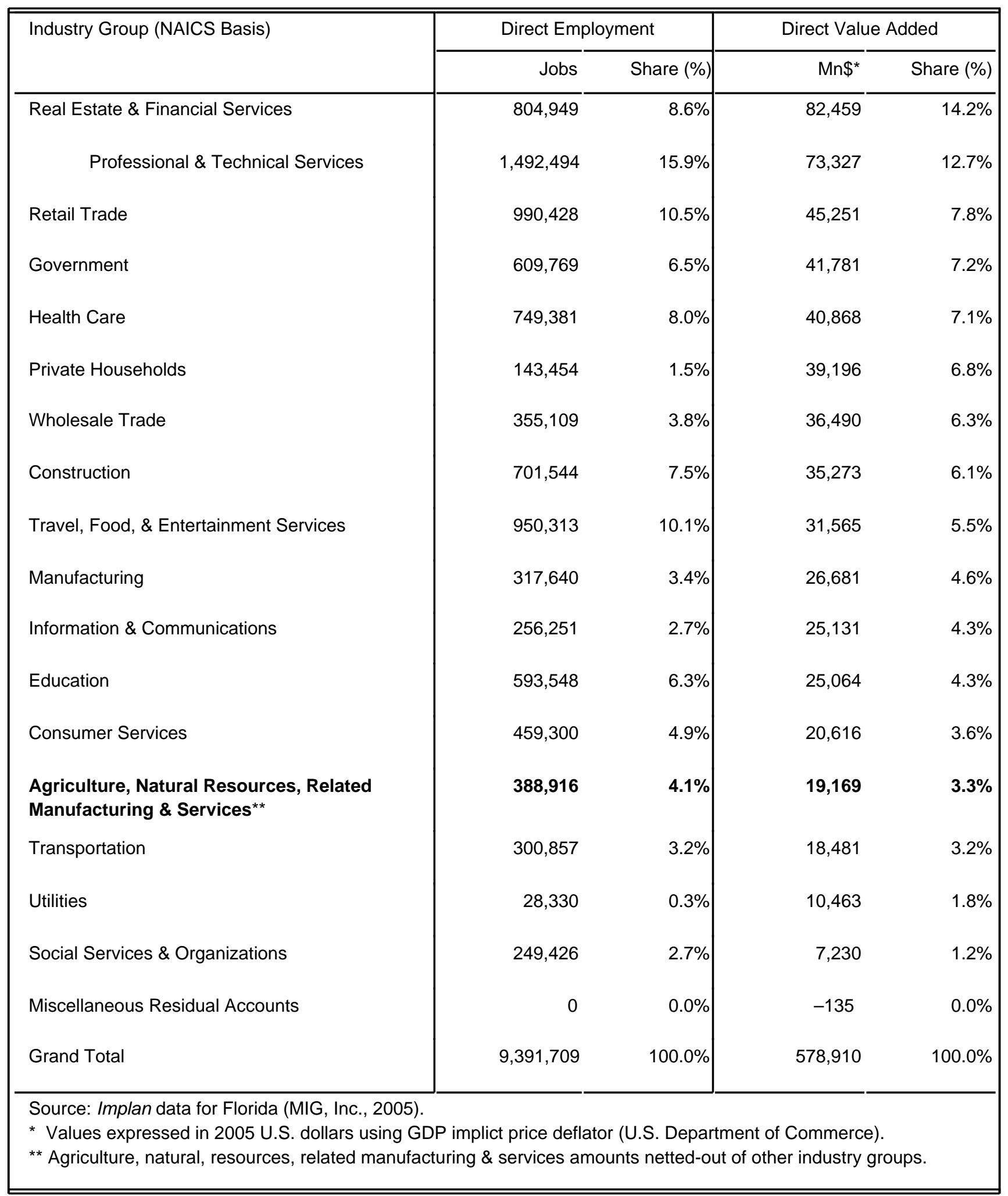

